\title{
NEXT GENERATION WIRELESS LAN SYSTEM DESIGN ${ }^{1}$
}

\author{
Chutima Prommak, Joseph Kabara, \\ David Tipper, Chalermpol Charnsripinyo
}

Department of Information Science \& Telecommunications

135 N. Bellefield ave., University of Pittsburgh, PA 15260

\begin{abstract}
An important issue in the widespread deployment of infrastructure based wireless local area networks (WLANs) is the network design. In this paper, we propose a new WLAN design approach that focuses on assuring sufficient data rate capacity to meet expected user demand in the coverage area, while still satisfying signal coverage and interference level requirements. Noting the low cost of WLAN access points, we formulate a novel mathematical network design model within the framework of constraint satisfaction problems. Our model is termed the capacity based WLAN constraint satisfaction problem (Cap-WLAN CSP). The solution of the Cap-WLAN CSP model yields a network design based on data rate demand by providing the access point locations, the frequency channel allocation, and power levels required for the WLAN to meet expected user demands. Our numerical results illustrate that the capacity based approach is more appropriate for the design of WLAN systems than those of traditional coverage based designs.
\end{abstract}

\section{INTRODUCTION}

Wireless local area networks (WLANs), such as those built on the IEEE $802.11 \mathrm{~b}$ standard, are experiencing tremendous growth, providing consumers and businesses mobile data networking capabilities that complement the mobile voice capabilities of cellular phones. The deployment of WLANs and the growth in the number of subscribers has been phenomenal and is expected to continue or increase at its fast pace [1-3, 13]. The fact that laptop computers and other mobile devices (e.g., PDAs) are becoming cheaper, smaller and more powerful has driven the demand for WLAN services. An important issue in such wide spread deployment of WLANs is the network design.

The network design determines the number, location and configuration (e.g., frequency, power level, etc.) of WLAN access points (APs) and the network capacity (aggregate bit rate) provided to a specific geographic area. In current practice, WLANs are largely designed in the basis of a trial and error, measurement based approach. Specifically, one places APs in buildings at opportunistic locations, measures the received signal strength in the desired coverage areas of the building and adjusts the AP locations, power levels, frequency channel etc., based on the observed coverage [15]. All of this is done manually, and is labor intensive. Such an approach is expensive and time consuming when deploying large numbers of WLAN APs.
In the research literature, work has appeared on developing algorithms for the design of WLANs in an indoor environment [4-6]. This work seeks to create a coverage based WLAN design; that is ensuring that an adequate received signal strength and signal-to-interference ratio (SIR) are maintained in the intended service area. These papers are similar in that they formulate optimal access point/ base station placement problems with very similar objective functions and vary only slightly in the assumptions and the approaches to solve the optimization problem. The current coverage based WLAN design approach is sufficient for small networks of a few APs where user density is low and traffic load is light. However, as the number of WLAN users and applications increases, network capacity becomes an issue and a fundamentally different approach to network design is required. A network design solution must account for wireless user density, expected user subscriber profiles, traffic models for various applications, and support for QoS classes. Support for these requirements in turn requires designing the WLAN system based on a data rate density criterion because the first step towards providing any kind of QoS is to ensure availability of the necessary bandwidth (data rate). However, current work in WLAN design ignores this issue and concentrates on providing signal coverage and acceptable interference levels in cells. While these factors play a crucial role in the overall design, they are not sufficient for guaranteeing a particular aggregate data rate capacity in a specific geographic area. In the recent literature Kabara[7] and Hills [8] discuss the need for considering of capacity requirements in WLAN design and Tutschku [11, 14] presents similar arguments for the consideration of traffic load in the design of circuit switched cellular voice networks.

In this paper, we present a new design methodology for infrastructure based WLANs capable of supporting a data rate demand (data rate density) in a given area. The design methodology will determine the number of access points (APs), frequency channels, power level and the placement of the APs, that will satisfy a set of constraints that include the data rate density requirement, radio propagation conditions and physical limitations like receiver sensitivity. Unlike current optimization approaches we formulate the design problem as a constraint satisfaction problem (CSP) [12] that we will refer to as Capacity based WLAN constraint satisfaction problem (Cap-WLAN CSP).

The remainder of the paper is organized as follows. Section II presents the formulation of the Cap-WLAN CSP and discusses a solution technique. Section III illustrates network

\footnotetext{
${ }^{1}$ Funded in part an NSF grant ANIR 9980516 and a NIST Critical Infrastructure Protection grant
} 
design results and discusses computational complexity. Finally, section IV concludes the paper.

\section{CONSTRAINT SATISFACTION PROBLEM FORMULATION}

The optimization based WLAN design approaches [4-6] aim to minimize the number of and optimize the location of the access points. This objective results in a very large and complex solution space and such design formulations are NP hard, thus heuristic sub-optimal solution techniques to the optimization models have been proposed. However, it is unnecessary in the design of WLAN systems to minimize the number of the APs due to the low cost of the AP compared to the wireless devices with which they are communicating. However, overprovisioning the service areas leads to serious system performance degradation due to co-channel interference. Thus, we propose that it is more appropriate and effective to formulate the design problem as a constraint satisfaction problem rather than an optimization problem. Our algorithm represents a service region as discrete space of grid size $1 \mathrm{~m} \times 1 \mathrm{~m}$. The grid points represent candidate locations to install APs and specify the locations that require radio signal coverage. In our design experiments we consider two cases, the first allows APs to be located at any grid intersection. The second restricts AP locations to a more narrowly defined feasible space (e.g., located only in hallways).

The general Cap-WLAN CSP design approach is shown in figure 1. The network design algorithm is structured into two main parts. The first part involves determining the minimum number of APs necessary for a given service requirement and initializing APs' configuration. The second stage implements a solution algorithm for the CSP and determines the AP parameters, including locations, power levels, and frequency channel, such that the designed WLAN system satisfies the service requirements. Two sets of input components are provided to the Cap-WLAN CSP algorithm. The first set of inputs defines the service environment including the spatial traffic demand distribution and the physical structure of the service area. The second set of inputs incorporates the path loss models that approximate radio propagation in the given physical service environment.

The Cap-WLAN CSP formulation is defined by the triple $(\mathbf{V}, \mathbf{D}, \mathbf{C})$, where $\mathbf{V}=$ the set of variables, $\mathbf{D}=$ the set of finite domains associated with the variables, and $\mathbf{C}=$ the set of constraints. We represent any two-dimensional space with $(x, y)$ coordinates. Let $A$ denote the set of $N$ access points $\left\{a p_{1}, a p_{2}, \ldots\right.$, $\left.a p_{n}\right\}, U$ denote the set of wireless users or demand nodes in the service area $\left\{u_{1}, u_{2}, \ldots, u_{m}\right\}, G C$ denote the set of grid points $\left\{g_{1}\right.$, $\left.g_{2}, \ldots, g_{c}\right\}$ representing the area that requires radio coverage.

We define a set of variables $\mathbf{V}=\left\{p_{j}, f_{j}, u_{i j}, g_{h j},\left(x_{j}, y_{j}\right)\right\}$ where $p_{j}$ is the power level of access point $j, \forall j \in A ; f_{j}$ is the frequency channel of access point $j, \forall j \in A ; u_{i j}$ is a binary variable that indicates whether user $i$ associates with access point $j$ or not, $\forall i \in U, \forall j \in A ; g_{h j}$ is a binary variable that indicates whether grid point $h$ can receive signal from access point $j$ or not, $\forall h \in G C, \forall j \in A ;\left(x_{j}, y_{j}\right)$ indicates the location of access points, $\forall j \in A$.

We also define the domains of the variables, $\mathbf{D}$ be the set of domains of the form $\left\{\mathbf{D}_{\mathbf{p}}, \mathbf{D}_{\mathbf{f}}, \mathbf{D}_{\mathbf{u i j}}, \mathbf{D}_{\mathbf{g h j}}, \mathbf{D}_{(\mathbf{x j}, \mathbf{y j})}\right\}$ where: $\mathbf{D}_{\mathbf{p}}$ $=$ the domain of $p_{j}$ variable for $\forall j \in A, \mathbf{D}_{\mathbf{f}}=$ the domain of $f_{j}$ variable for $\forall j \in A, \mathbf{D}_{\text {uij }}=$ the domain of $u_{i j}$ variable $=\{0,1\}$ for $\forall i \in U, \forall j \in A, \mathbf{D}_{\mathbf{g h j}}=$ the domain of $g_{h j}$ variable $=\{0,1\}$ for $\forall h \in G C, \forall j \in A, \mathbf{D}_{(\mathbf{x j}, \mathbf{y j})}=$ the domain of $\left(x_{j}, y_{j}\right)$ variable $=\left\{x_{\text {min }}\right.$ $<x_{j}<x_{\max }$ and $\left.y_{\min }<y_{j}<y_{\max }\right\}$ for $\forall j \in A$.

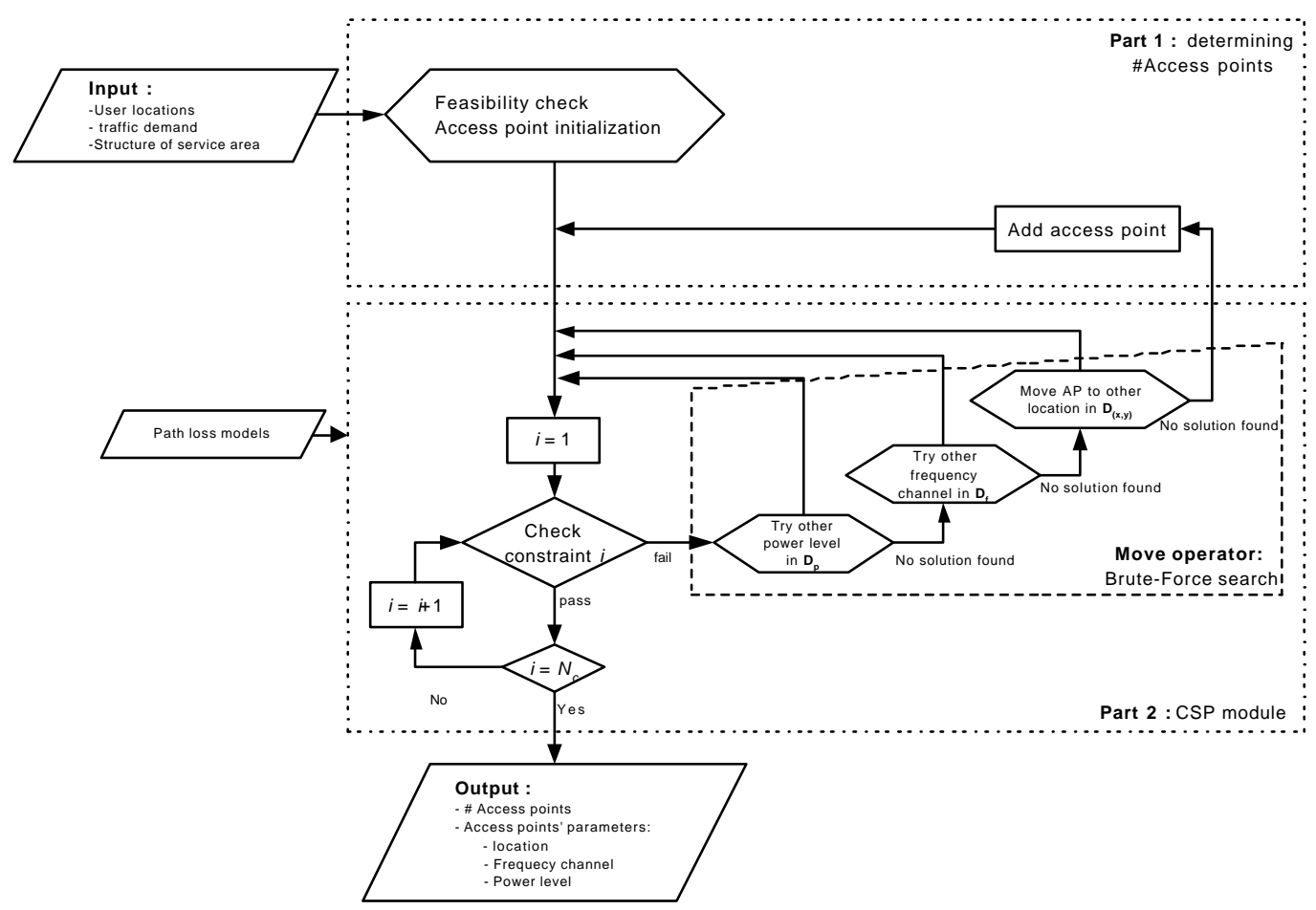

Figure 1 Cap-WLAN CSP Algorithm 
We define a set of constraints $\mathbf{C}=\{\mathbf{C 1}, \mathbf{C 2}, \mathbf{C 3}, \mathbf{C 4}$, C5, C6 $\}$. Each constraint put restrictions and requirements to the WLAN design as follows: C1 states that each wireless terminal is associated to one access point. C2 states that the signal received at each wireless terminal must be greater than the receiver threshold sensitivity. $\mathbf{C 3}$ assures that the traffic demand of wireless terminals assigned to a particular AP does not exceed the data rate capacity of the AP. Here, we incorporate the effective capacity coefficient $(\beta)$ to capture the effects of capacity reduction due to the number of wireless terminals and wireless terminals' traffic characteristics associated to the AP. C4 specifies the interference threshold of the wireless terminal. C5 states that a portion of mean data rate from all wireless users in a service area is served by available APs. $\alpha$ specifies a portion of all traffic demand that we consider serving. C6 states that the radio signal will be available across the specified coverage space. This condition allows the grid point to be able to receive radio signal from more than one access points, i.e. it allows over-lapping of the access points' coverage areas. $g_{h j}=1$ if the received signal strength at the grid point $h$ from the access point $j$ is greater than $P_{R}$ and the associated interference level is below $P_{I} ; g_{h j}=0$ otherwise. Note that constraint C1, C2, C4 and C6 include the path loss model, which may vary for different service area environments. To solve the Cap-WLAN CSP, we employ a brute force search technique, where variable values are tested in sequence. In the next section we demonstrate that a brute-force search is in some case efficient enough.

\section{NUMERICAL RESULTS}

\section{A. 802.11b design example}

Numerical network design experiments were conducted for a small and large single-floor service area with low and high data rate requirement scenarios. The small service area is the fourth floor $(21 \mathrm{~m} \times 35 \mathrm{~m})$ of the Information Science (IS) building at the University of Pittsburgh. The large service area is the first floor $(60 \mathrm{~m} \times 65 \mathrm{~m})$ of the Hillman library at the University of Pittsburgh. We classify wireless users into three categories based on typical demands in this environment. Those with handheld computers (smaller devices with lower computational power, smaller memory like an HP Jornada) access the network at $50 \mathrm{Kbps}$. The second class of wireless devices need a medium data rate of $250 \mathrm{Kbps}$ as they employ laptops to mostly read email and access some Internet sites with little multimedia content. The third category consists of high data rate wireless terminals who use their laptops at the fullest network speed possible (current measurements indicate that typical laptops transmit or receive data at about $2 \mathrm{Mbps}$ ). These wireless terminals utilize remote file systems and streaming audio/video.

The domains for the variables were tailored to current 802.11 b practices; $\mathbf{D}_{\mathbf{p}}=\{15,20,24\}$ in $\mathrm{dBm}$, and $\mathbf{D}_{\mathbf{f}}=\{2.412$, $2.437,2.462\}$ in $\mathrm{GHz}$. These experiments employed the log distance path loss model [10] to estimate radio propagation characteristics. Input parameters were selected with respect to the service environment, the building structure and the $802.11 \mathrm{~b}$ specification. Here, $n_{0}=3.02$ [10], $K_{\sigma}=10[10], P_{R}=-80 \mathrm{dBm}$, $P_{I}=-90 \mathrm{dBm}$, and $C_{a p}=11 \mathrm{Mbps}$ [9]. The design aims to serve
Figure 2 Constraint Satisfaction Problem Formulation: 2-D capacity-based WLAN design

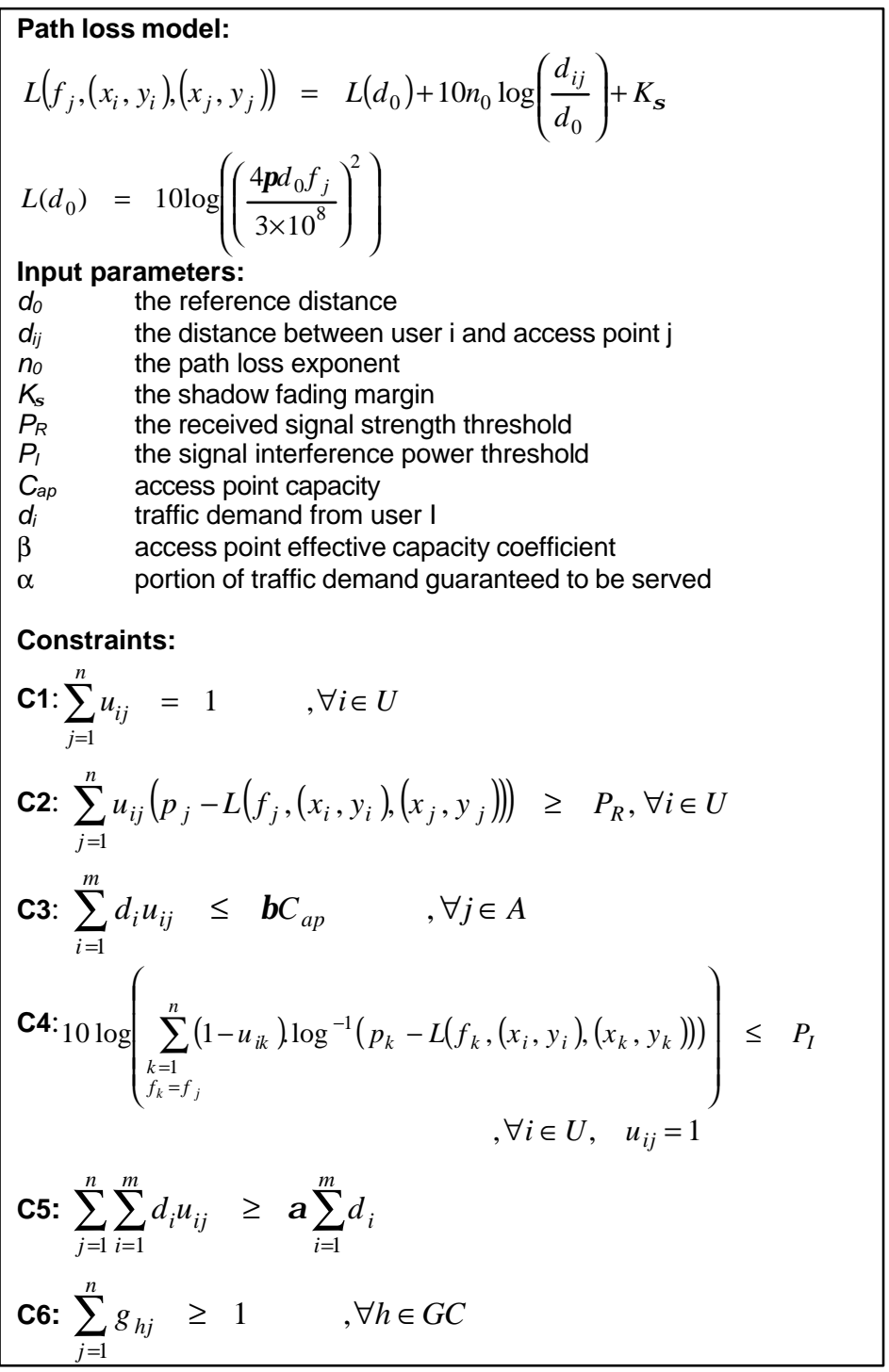

all traffic demand, i.e., $\alpha=1$ on a $1 \mathrm{~m} \times 1 \mathrm{~m}$ grid. Due to the contention based MAC protocol, it is assumed that the access points' throughput reduces to $90 \%$ of the access point full capacity, i.e. $\beta=0.9$.

Figure 4 illustrates the case of the small service area with a coverage-based design. In this example the WLAN system utilizes two access points to cover the entire service areaat the specified signal strength threshold, given the propagation environment. However, this design does not provide adequate capacity to all wireless users. In this network there are a total of 33 users, 23 users require $250 \mathrm{Kbps}$ each and 10 users require $2 \mathrm{Mbps}$ each, this results in total traffic demand of 25.75 Mbps. Hence, the use of two access points can not support all traffic load. For this same service scenario, however, the CapWLAN CSP design approach estimates the number of APs from the aggregate traffic demand, which in this case requires a minimum of three APs. Then a brute-force search algorithm determines access points' parameters, including locations, power levels, and frequency channels as shown in figure 5. 
Another example of the small service area illustrates the more complex case where we consider a higher user density and traffic demand as depicted in figure 6. 68 wireless users are classified as 16 of $50 \mathrm{Kbps}$ wireless terminals, 38 of $250 \mathrm{Kbps}$ wireless terminals, and 14 of 2 Mbps wireless terminals. In this case a minimum number of four APs are required. The solution for this case assigns different power levels to access points resulting in different coverage size as depicted in figure 6 . The majority of low bit rate users are covered by access point AP1. In the left and top right corners of the service area, small groups of users demanding much higher data rate are served by smaller coverage of AP0, AP2, and AP3.

The effects of density and distribution of wireless users on the WLAN system infrastructure are more obvious in large service areas. Consider the design of the large service area with the light and heavy load scenarios. Figure 7 and 8 show different system configurations for both scenarios. Again for the light load scenario, the design is comparable to the coverage-based design where sufficient signal strength is provided across the service region. We can observe that the coverage-based design is not suitable for heavy load scenarios, whereas the capacity-based design approach taking into account user density and traffic load yields an effective system configuration for the high traffic load environment.

\section{B. Computational Requirements}

The brute-force search algorithm is simple to implement but in the worst case, the whole solution space must be searched exhaustively. Consider the complexity of the CSP module for the problem with $n$ number of variables, $e$ number of constraints, and each variable consists of $a$ candidate values. There are altogether $a^{n}$ possible combinations (candidate solutions) of ntuples. Thus, the complexity of the exhaustive-search algorithm is $O\left(e a^{n}\right)$. We can see that the complexity of the CSP module increases exponentially with the number of variables $n$. Table 1 shows computational times running on a $350 \mathrm{MHz}$ Sun Ultrasparc II proces sor to solve the WLAN design for the small service area with various user density and traffic scenarios by the brute-force approach. Figure 3 depicts the rising of the computational time as the number of wireless users and the traffic load increase. We see that the computation time rises to more than 5000 seconds for even a moderate, 90, number of users. However, as discussed earlier, we also considered the case of a restricted set of locations for the AP. In this case we see that when we restrict AP locations to lay within the hallway area, which are often desirable locations because of proximity to power and wired network infrastructure. We see that by restricting the space even the brute force search time, while growing is only 1 second for a 90 user space.

Table 1 Computational time of brute-force search

\begin{tabular}{|c|c|c|c|c|}
\hline $\begin{array}{c}\text { \# Demand } \\
\text { nodes in } \\
\text { service area }\end{array}$ & $\begin{array}{c}\text { Aggregate } \\
\text { traffic demand }\end{array}$ & \multirow{2}{*}{$\begin{array}{c}\text { \# Access } \\
\text { points }\end{array}$} & \multicolumn{2}{|c|}{ CPU time (sec) } \\
\cline { 4 - 5 } & required & $\begin{array}{c}\text { Search the } \\
\text { entire area }\end{array}$ & $\begin{array}{c}\text { Search along } \\
\text { the wall }\end{array}$ \\
\hline 7 & 5.25 & 2 & 0.004 & 0.004 \\
\hline 15 & 16.00 & 2 & 0.006 & 0.006 \\
\hline 30 & 25.75 & 3 & 0.040 & 0.039 \\
\hline 45 & 28.50 & 3 & 0.058 & 0.047 \\
\hline 60 & 34.95 & 4 & 700 & 0.851 \\
\hline 75 & 38.85 & 4 & 1904 & 0.911 \\
\hline 90 & 39.60 & 4 & 5256 & 1.113 \\
\hline
\end{tabular}

\section{CONCLUSIONS}

In this paper we propose a new WLAN design strategy called capacity based WLAN design. The problem is formulated as a constraint satisfaction problem, which can guarantee not only radio coverage to the target service area but also provide a specified data rate capacity to carry the traffic demand from each user in the service area. We conducted several design experiments, which illustrate the benefits of the capacity-based WLAN design approach over the traditional coverage-based design. Currently, by limiting the search space to the most desirable locations even a brute-force search technique succeeds in reasonable time.

\section{ACKNOWLEDGMENT}

We gratefully acknowledge Prashant Krishnamurthy for his thoughtful and constructive comments.

\section{REFERENCES}

[1] US News and World Report.pp. 48-58. Dec13, 1999.

[2] J. T. Geier, Wireless LANs, 2 ed. Indianapolis : Prentice Hall, 2001.

[3] A. Santamaria, and F. J., Lopez-Hernandez, Wireless LAN standards and applications Boston: Artech House, 2001.

[4] M. Unbehaun, "Coverage planning for indoor wireless LAN systems, ," http://www.s3.kth.se/ matthias/Public/ Stanford/MatthiasUnbehaun.Abstract.pdf., June2001

[5] D. Stamatelos and A. Ephremides, "Spectral efficiency and optimal base placement for indoor wireless networks," IEEE Journal on selected areas in communications, vol. 14, no. 4, pp. 651-661, May1996.

[6] R. C. Rodrigues, G.R. Mateus, and A.A. F. Loureiro, "On the design and capacity planning of a wireless local area network," IEEE Conference on Network Operations and Management Symposium, pp. 335-348, 2000.

[7] J. Kabara, P. Krishnamurthy, and D. Tipper, "Capacity based network planning for wireless data networks," Proceedings of IST Mobile Communications Summit, Sept.1

[8] A. Hills, "Large-scale wireless LAN design," IEEE Communication Magazine, vol. 39, no. 11, pp. 98-104, Nov.2001.

[9] IEEE-SA Standards Board. Part 11: Wireless LAN Medium Access Control (MAC) and Physical Layer (PHY) specifications: Higher-speed physical layer extension in the $2.4 \mathrm{GHz}$ band. 1999.

[10] T. S. Rappaport, "Mobile radio propagation: Large-scale path loss," Wireless communications: Principles \& Practice, 1996, pp. 69-138.

[11] K. Tutschku and P. Tran-Gia, “Spatial Traffic Estimation and Characterization for Mobile Communication Network Design," IEEE Journal on Selected Areas in Communications, Vol. 16, No. 5., pp. 804-811, June, 1998

[12] Z. Ruttkay, "Constraint Satisfaction - a Survey," CWI Quarterly, vol. 11, no. 2-3, pp. 163-214, 1998.

[13] J.Kabara and D.Tipper, "'A Design Tool for Wireless Local Area Networks,' Pittsburgh Digital Greenhouse, April, 2002

[14] K. Tutschku, "Demand-based radio network planning of cellular mobile communication systems, " INFOCOM 98, vol. 3 pp. 1054-1061, 1998

[15] B.J. Bennington and C.R. Bartel, "Wireless Andrew: Building a high speed campus-wide wireless data network", Mobile Networks and Applications, vol. 6 pp.9-22, 2001. 


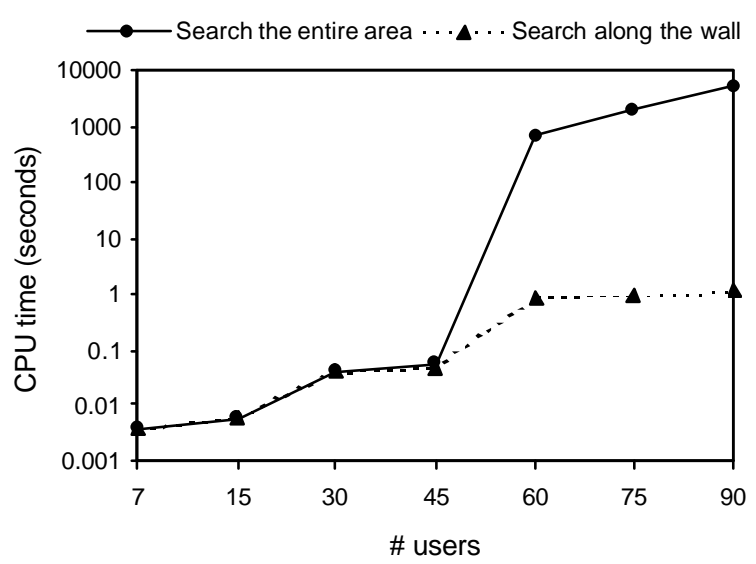

Figure 3 Exponentially computational time of bruce-force search approach

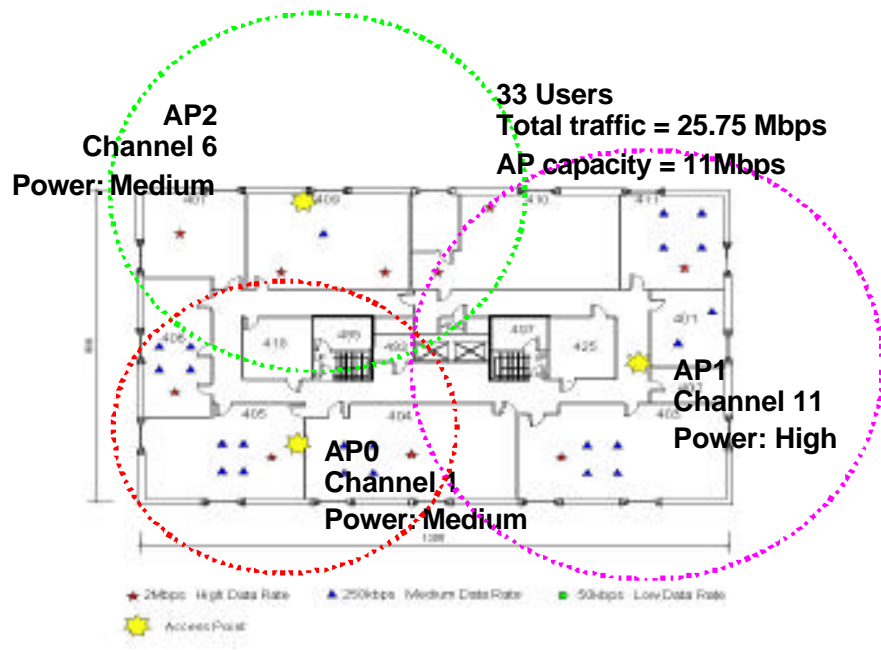

Figure 5 Small service area using capacity-based design

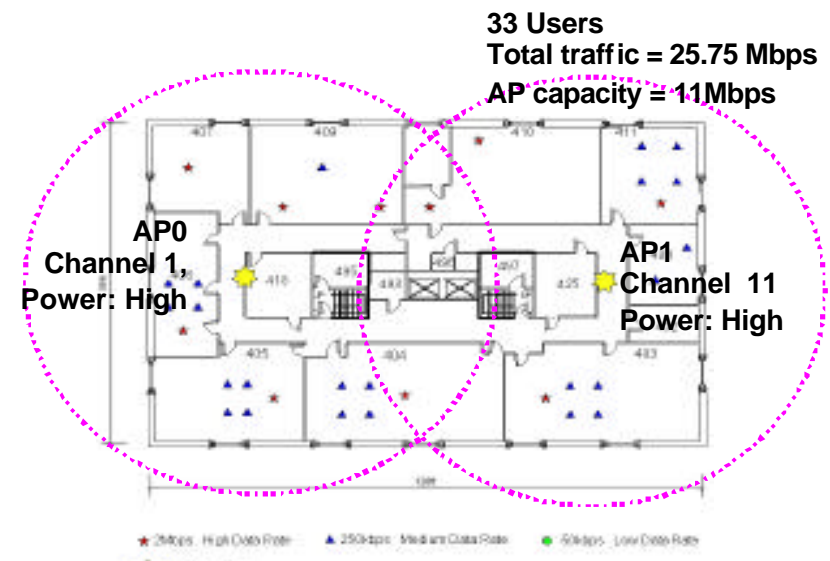

Figure 4 Small service area using coverage-based design

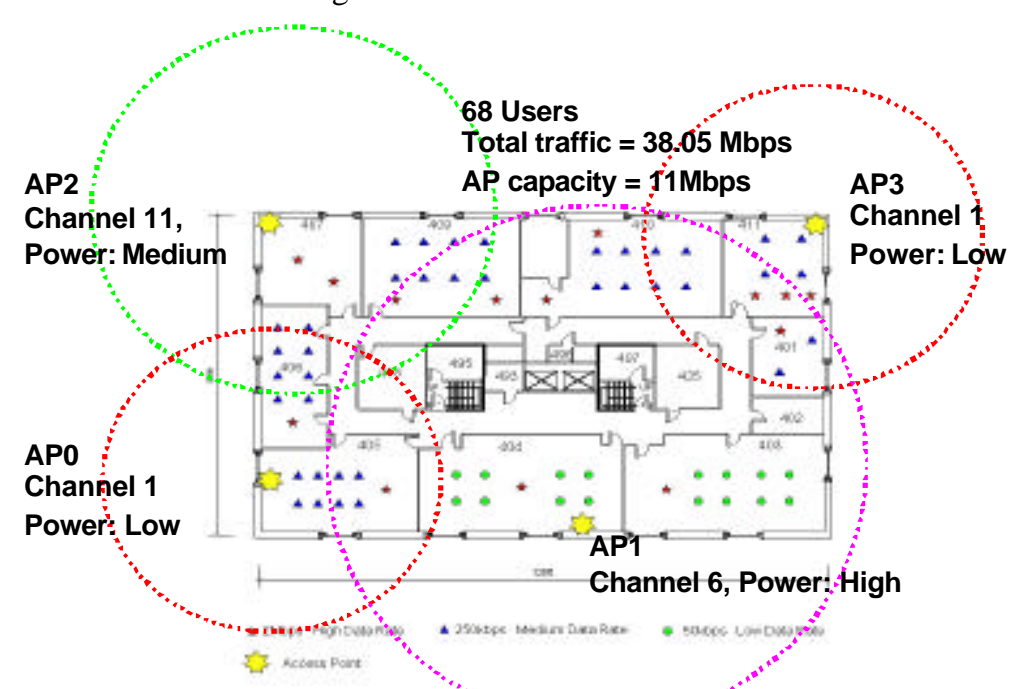

Figure 6 Small sèrvice area with heavy load using capacity-based design

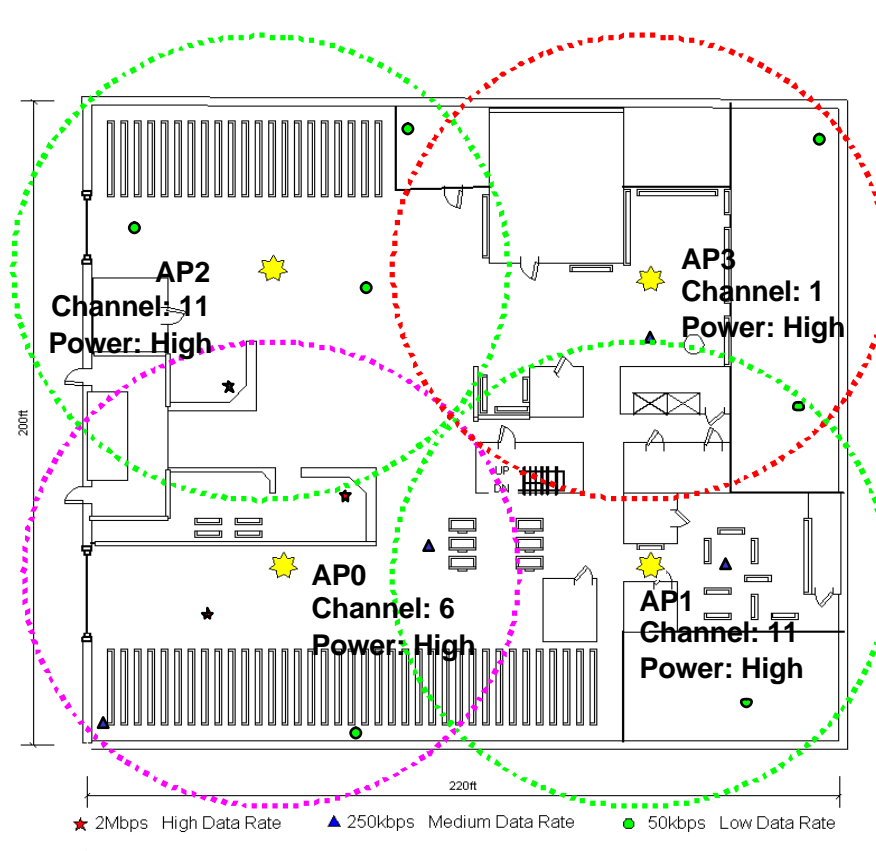

$\sum$ Access Point

Figure 7 Large service area, light load using capacity-based design

(Comparable to coverage-based design)

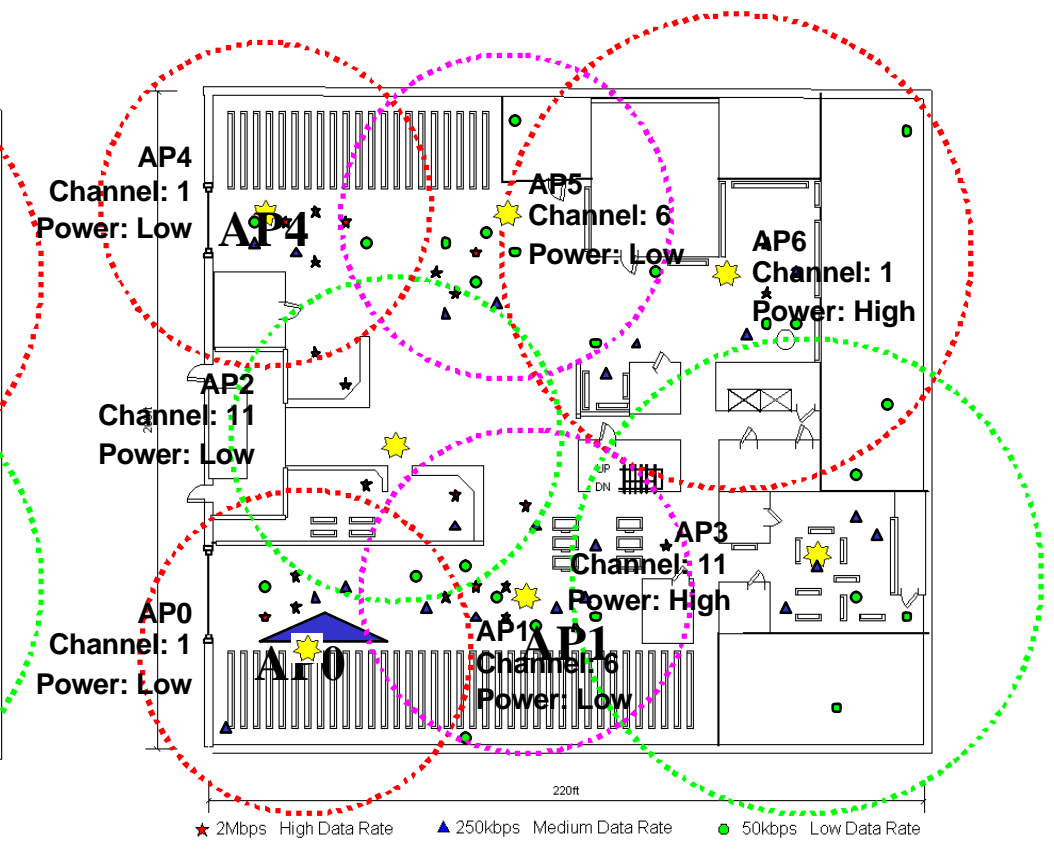

Figure 8 Large service area, heavy load using capacitybased design 